\title{
Magnetic Water and Chemical Substances Impact on Growth Parame- ters and Yield Components of Onion Crop under Assiut Conditions
}

\author{
S.Y. Attalah \\ Department of Vegetable Crops, Faculty of Agriculture, Assiut University, Assiut, Egypt \\ Received on: $2 / 2 / 2017$ \\ Accepted for publication on: 15/2/2017
}

\begin{abstract}
The experiments of this study were conducted to investigate the effect of magnetized water and two other chemical substances thatare kown as Agro promotor1 (substance No.48) and Agro promotor2 (substance No.50) on growth, yield and chemical composition of onion variety Giza 6. The sesubstances were obtained from Cairo University. Result revealed that growth parameters, yield and its component and chemical contents increased with using both of magnetic water and Agro promotor1 (substance No.48) at concentration of $3 \mathrm{ml} / 1$ comparing with control (untreated) treatment. Using magnetized water and Agro promotor1 (substance No.48) at concentration of $3 \mathrm{ml} / 1$ induced positive significant effect on plant height and weight, number of leaves/plant and bulb diameter as well as significantly improved neck and bulb diameters, bulb weight, total soluble soiled, total yield and marketable yield percentage than plants of control treatment which showed an increase in percentage of bolters, in both season. In general, it might be concluded that application of each of magnetized water and Agro promotorl (substance No.48) treatments proved to be good technology to enhance growth, yield and quality when compare with untreated treatment. The marketable yield was increased by $30 \%$ as compared with control treatment by using magnetized water and increased by $22-235 \%$ when substance No.48 at concentration of $3 \mathrm{ml} / 1$ was used. The increase in total yield ranged from 12$15.4 \%$ when using magnetized water and was $14-15.4 \%$ when substance No.48 at concentration of $3 \mathrm{ml} / 1$ compared with control treatment. This study recommends using magnetized water to obtain the highest marketable yield and the lowest percentage of bolters. In case of magnetized water un-available, Agro promotor 1 (substance No.48) at concentration of $3 \mathrm{ml} / \mathrm{l}$ can be applied.
\end{abstract}

Keyword: bulbing ratio, bolters, chemical content, marketable yield, TSS, yield quality.

\section{Introduction}

Onion (Allium cepa L.) is one of the most important vegetable crops grown in the world. In Egypt, it is ranked as the third important vegetable crops after tomato and potato according to annual production (2024881 ton) FAOSTAT (2012). Utilization of magnetized water technology is considered as a promising technique to improve water use efficiency and crop productivity. The ir- rigation by magnetized water increased significantly plant height, number of leaves / plant, fresh and dry weight, as well as survival rate, $\mathrm{N}$ and $\mathrm{P} \%$ than those irrigated by nonmagnetized water on pear seedlings (Osman et al., 2014) and in tomato (Carbonell, 2011 and Ahmed, 2013). Magnetized water increases plant metabolism in terms of photosynthesis and water uptake (Yano et al., 2004). Magnetized water improved plant 
stimulation of synthesis and transport of hormones and enzymes metabolism and increase the final plant yield (Esitken, 2003).

Water molecule consists of hydrogen and oxygen atoms are partly positive and partly negative that forming by weak attraction allowing to the formation of hydrogen bonds. The magnetic and electrical fields are extremely affected in liquid water through hydrogen bond that changes some physical and chemical properties of magnetic water (Nasher, 2008). The magnetic field on ion of positive charge will create magnetic force and move in the direction relative to the right hand rule of Lorantz, while the negative charge particle moved in the opposite direction (Amer et al, 2006). The motion in the direction of water molecules charges will increasing the velocity of the particles that create more collisions between the particles (Gholizadeh etal., 2008). The magnetic field increases the strength of hydrogen bond, which leads to increasing in the refractive index.

Currently, magnetized water is used to increase plant yields (Lin and Yotvat, 1990), many benefits of human health and change in $\mathrm{pH}$ of the water (Maheshwari, 2009; Kordas, 2002). The magnetic water treatment can improve acceleration of seeds metabolism and increased yield parameter of the crops such as cereal sunflower and soybean (Özalpan et al., 1999; Yurttas et al., 1999; Oldacay, 2002). Magnetic treated water undergoes several changes in its physical properties. It also exerts several effects on the soil-water-plant system. Leaching the soil with MW significantly increases available soil phosphorus content compared with the leaching with normal water at all soil depths. Behavior of nutrients under an MF is a function of their magnetic susceptibility (ALI et al., 2014). Therefore, the aim of this research work was to assess response of onion plants Giza $6 \mathrm{cv}$. to magnetized irrigation water and other two chemical substances i.e., Agro promotor1 (substance No.48) and Agro promotor2 (substance No.50) that were obtained from Cairo university to enhance growth, yield and quality.

\section{Materials and Methods}

The field work of this study was carried out at the Experimental Farm of Faculty of Agriculture, Assiut University, Assiut, Egypt, during 2014/2015 and 2015/2016 winter seasons. The experiments were conducted to study the response of onion plants Giza $6 \mathrm{cv}$. grown in clay soil to magnetized irrigation water and other two substance i.e., Agro promotor1 (substance No.48) and Agro promotor2 (substance No.50) that were obtained from Cairo university. The component of substance, 48 is (sodium chloride $0.28 \%$, organic matter $0.02 \%$, powder of rock salts $0.01 \%$, hypochlorite and hypochloros acid $0.05 \%$, electo-magnet activated water $5 \%$ and electro-magnet activated water till $100 \mathrm{ml}$. While the component of substance, 50 is (sodium chloride $0.28 \%$, organic matter $0.02 \%$, powder of rock salts $0.01 \%$, hypochlorite and hypochloros acid $0.05 \%$, and electromagnet activated water till $100 \mathrm{ml}$. These components according to (Kaoud, 2014). Six treatments were used which were magnetic water (MW), substance No.48,1.5 ml/1, sub- 
stance No.48,3 $\mathrm{ml} / 1$ substance No.50,1.5 ml/1, substance No.50,3 $\mathrm{ml} / \mathrm{l}$ and non-magnetized water (NMW) (un treated control). Onion seedlings were planted on $1^{\text {st }}$ and $4^{\text {th }}$ of September in both growing seasons respectively. Treatments were applied in September, 23 and 27 respectively as first time then at 15 dintervals for six times. All agricultural practices for onion crop production were applied as recommended for onion production (Hassan, 1991). The experiment was conducted using three replications in randomized complete-block design. Each experimental plot consisted of three rows. Each row was 3.5 meters long and 70 $\mathrm{cm}$ wide. Planting distance were 5-7 $\mathrm{cm}$ apart. Onion seedlings were sown on two side of ridge. Three samples were taken. First sample was taken after 30 days from treatments application, then at 30 d-intervals. Five plants of each replicate were randomly taken for recording vegetative growth characteristics, i.e., plant height $(\mathrm{cm})$, (plants was measured from the bulb base to the tip of the leaf blade), plant weight, number of leaves/plant, neck diameter $(\mathrm{cm})$ and bulb diameter $(\mathrm{cm})$. Bulbingratio was measured according to Manns (1952), using the following formula.

\section{Bulbing ratio $=$ Neck diameter $(\mathrm{cm}) /$ Bulb diameter (cm)}

Yield parameters and its components: At harvesting stage (150 days from transplanting date), a sample of 10 onion plants randomly taken from each experimental plot fordetermining yield characteristics, i.e., neck and bulb diameter $(\mathrm{cm})$, bulbing ratio, and average bulb fresh weight. In addition, total yield (ton/fed.) and percentage of marketable yield, and flowering plants (bolters) were measured.

Experimental design and statistical analysis: Data from each season, separately, were subjected to analysis of variance according to Snedecor and Cochran (1980). Based on homogeneity of error variance, the two seasons combined data were used in combined analysis of variance. Means of the treatments were compared using the Least Significant Difference (LSD) test at 0.05 probability level.

\section{Results and Discussion}

The obtained data present in Table 1, 2 and 3 revealed that there were significantly differences between treatments. Using of magnetized water, Agro promotor1 (substance No.48) and Agro promotor2 (substance No.50) induced positive effect on growth and yield parameters as comparing with control treatments.

Magnetized water treatment gave the proper values for bulb and neck diameter than chemical substance treatments (Table 3). Magnetized water treatment also increased marketable yield by $30 \%$ and increased total yield by $12-15,4 \%$ as compared with control treatment (Table 4). Using of magnetized water reduced percentage of bolters and increase total soluble soiled (Table 4).

The stimulatory effect of the application of magnetic water on the growth parameters reported in this study may be attributed to the increase in photosynthetic pigments, endogenous promoters (IAA) (Fomicheva et al. 1992 a \& b). Also, Belyavskaya (2001) reported that magnetic water significantly induces 
cell metabolism and mitosis meristematic cells of pea, lentil and flax, as well as stimulated synthesis and transport of hormones and enzymes metabolism and increased growth (Esitken 2003).

According to the response of chemical substance to growth parameters and yield components, Agro promotor1 (substance No.48) was superior to Agro promotor2 (substance No.50) as it gave proper value for percentage of marketable yield and total yield (ton/fed) and gave lower percentage of bolters than Agro promotor2 (substance No.50) (Table 4). Agro promotor1 (substance No.48) accelerating growth and yield parameters as it increased plant height, plant weight, number of leaves per plant, bulb diameter and decreased neck diameter (Table 1,2 and 3).

Using different concentrations i.e 1.5 and $3 \mathrm{ml} /$ linduced significant effect on growth parameter such as, plant height, plant weight and number of leaves/plant. Also different concentrations had significant effect on yield and its components such as, bulb and neck diameter, bulbing ratio, bulb weight, percentage of marketable yield, and percentage of bolters.

Application of Agro promotor1 (substance No.48) at concentration of $3 \mathrm{ml} / 1$ gave higher values for plant height, plant weight, number of leaves per plant than using of Agro promotor1 (substance No.48) at concentration of $1.5 \mathrm{ml} / \mathrm{l}$.
According to bulb and yield parameter, using of Agro promotor1 (substance No.48) at concentration of $3 \mathrm{ml} / 1$ lead to increase in bulb weight and percentage of marketable yield than using of Agro promotorl (substance No.48) at concentration of 1.5 $\mathrm{ml} / \mathrm{l}$. Percentage of marketable yield was increased by $22-23 \%$, while total yield was increased by $14-15.4 \%$ as compared with control treatment (Table 4). However, there was no significant effect on total yield and total soluble soiled when using either Agro promotor1 (substance No.48) at concentration of 1.5 or $3 \mathrm{ml} / 1$ (Table 4 ).

It was quite evident from Table 4 that, using of both magnetized water and Agro promotor1 (substance No.48), significantly improved neck and bulb diameters, bulbing ratio, bulb weight and total soluble soiled, total yield and marketable yield percentage than plants of control treatment, which was increased flowering bulb percentage. Obtained results agreed with those found by (Hozayn 2010; Ahmed 2013 and Rawabdeh et al, 2014).

\section{Conclusion}

In general, it might be concluded that irrigation with magnetized water proved to be good technology to enhance growth, yield and quality when compare with nonmagnetized water. When magnetized water is not available, we can use Agro promotor1 (substance No.48) at concentration of $3 \mathrm{ml} / 1$. 
Table 1. Growth parameters after 30 days from applying treatments (first sample) as affected by magnetic water and chemical substances during 2014-2015 and 2015-2016 seasons ${ }^{(1)}$

\begin{tabular}{|c|c|c|c|c|c|c|c|}
\hline \multicolumn{2}{|c|}{ Treatments } & $\begin{array}{c}\text { Bulb } \\
\text { diameter } \\
(\mathrm{cm})\end{array}$ & \begin{tabular}{|c|} 
Neck diame- \\
ter \\
$(\mathrm{cm})$
\end{tabular} & $\begin{array}{l}\text { Plant } \\
\text { height } \\
(\mathrm{cm})\end{array}$ & $\begin{array}{c}\text { Plant } \\
\text { weight } \\
\text { (g) }\end{array}$ & $\begin{array}{l}\text { Bulbing } \\
\text { ratio } \\
\text { (cm) }\end{array}$ & $\begin{array}{c}\text { No. of } \\
\text { leaves/pl. }\end{array}$ \\
\hline & & \multicolumn{6}{|c|}{ 2014-2015 } \\
\hline \multicolumn{2}{|l|}{ "48,(1.5 cm/l) } & $2.48 \mathrm{~b}$ & $1.44 \mathrm{~d}$ & $54.63 \mathrm{~b}$ & $80.533 \mathrm{c}$ & $0.581 \mathrm{~d}$ & $12.06 \mathrm{c}$ \\
\hline \multicolumn{2}{|l|}{$48,(3 \mathrm{~cm} / \mathrm{l})$} & $2.82 \mathrm{a}$ & $1.26 \mathrm{f}$ & $57.46 \mathrm{a}$ & $86.067 \mathrm{a}$ & $0.447 \mathrm{f}$ & $14.33 \mathrm{a}$ \\
\hline \multicolumn{2}{|l|}{$50,(1.5 \mathrm{~cm} / \mathrm{l})$} & $2.40 \mathrm{c}$ & $1.57 \mathrm{~b}$ & $50.63 \mathrm{c}$ & $70.233 \mathrm{e}$ & $0.654 \mathrm{~b}$ & $10.8 \mathrm{e}$ \\
\hline \multicolumn{2}{|l|}{$50,(3 \mathrm{~cm} / \mathrm{l})$} & $2.43 \mathrm{bc}$ & $1.49 \mathrm{c}$ & $51.43 \mathrm{c}$ & $73.500 \mathrm{~d}$ & $0.613 \mathrm{c}$ & $11.26 \mathrm{de}$ \\
\hline \multicolumn{2}{|l|}{${ }^{* * * *} \mathbf{M ~ w}$} & $2.85 \mathrm{a}$ & $1.36 \mathrm{e}$ & $56.33 \mathrm{ab}$ & $83.267 \mathrm{~b}$ & $0.475 \mathrm{e}$ & $13.00 \mathrm{~b}$ \\
\hline \multicolumn{2}{|l|}{ Control } & $2.33 \mathrm{~d}$ & $1.62 \mathrm{a}$ & $51.26 \mathrm{c}$ & $70.530 \mathrm{e}$ & $0.697 \mathrm{a}$ & $11.62 \mathrm{dc}$ \\
\hline & & \multicolumn{6}{|c|}{ 2015-2016 } \\
\hline \multicolumn{2}{|l|}{$48,(1.5 \mathrm{~cm} / \mathrm{l})$} & $2.49 \mathrm{bc}$ & $1.46 \mathrm{c}$ & $55.46 \mathrm{~b}$ & $81.73 \mathrm{~b}$ & $0.584 \mathrm{~d}$ & $12.43 \mathrm{c}$ \\
\hline \multicolumn{2}{|l|}{$48,(3 \mathrm{~cm} / \mathrm{l})$} & $2.83 \mathrm{a}$ & $1.25 \mathrm{e}$ & $58.30 \mathrm{a}$ & $84.56 \mathrm{a}$ & $0.443 \mathrm{f}$ & $14.66 \mathrm{a}$ \\
\hline \multicolumn{2}{|l|}{$50,(1.5 \mathrm{~cm} / \mathrm{l})$} & $2.50 \mathrm{~b}$ & $1.55 \mathrm{~b}$ & $52.30 \mathrm{c}$ & $71.16 \mathrm{~d}$ & $0.619 \mathrm{~b}$ & $11.43 \mathrm{e}$ \\
\hline \multicolumn{2}{|l|}{$50,(3 \mathrm{~cm} / \mathrm{l})$} & $2.45 \mathrm{c}$ & $1.47 \mathrm{c}$ & $52.03 \mathrm{c}$ & $74.86 \mathrm{c}$ & $0.601 \mathrm{c}$ & $12.23 \mathrm{~cd}$ \\
\hline \multicolumn{2}{|l|}{ M w } & $2.87 \mathrm{a}$ & $1.36 \mathrm{~d}$ & $57.10 \mathrm{ab}$ & $84.03 \mathrm{ab}$ & $0.475 \mathrm{e}$ & $13.33 \mathrm{~b}$ \\
\hline \multicolumn{2}{|l|}{ Control } & $2.31 \mathrm{~d}$ & $1.65 \mathrm{a}$ & $51.76 \mathrm{c}$ & $73.43 \mathrm{~cd}$ & $0.715 \mathrm{a}$ & $11.53 \mathrm{de}$ \\
\hline $\begin{array}{l}\text { Source of } \\
\text { variation }\end{array}$ & d.f. & \multicolumn{6}{|c|}{ Mean Square } \\
\hline Year & 1 & 0.00613 & 0.00006944 & 6.76000 & 8.02777 & 0.000220 & 1.62137778 \\
\hline $\begin{array}{l}\text { Rep(within } \\
\text { year) }\end{array}$ & 4 & 0.0060 & 0.00017778 & 3.1105556 & 4.040278 & 0.000355 & 1.1074888 \\
\hline Treat & 5 & $0.30401 *$ & $0.11031611^{*}$ & $49.51577^{*}$ & $238.9837^{*}$ & $0.05886^{*}$ & 9.3208000* \\
\hline $\begin{array}{l}\text { Treat } x \\
\text { year }\end{array}$ & 5 & $0.00229 *$ & $0.00055611^{*}$ & $\begin{array}{c}0.257333 \\
\text { (N.S.) }\end{array}$ & $3.022444^{*}$ & $0.00046^{*}$ & $0.1856444 *$ \\
\hline Error & 20 & 0.000782 & 0.00012444 & 1.3528889 & 2.043611 & 0.000066 & 0.1466222 \\
\hline
\end{tabular}

${ }^{(1)}$ Means within column followed by the same letter(s) are not significantly different at 0.05 level of probability.

*Agro promotor1 (substance No.48).

*** Agro promotor2 (substance No.50).

${ }^{* * *}$ MW (Magnetic water) 
Table 2. Growth parameters after 60 days from applying treatments (second sample) as affected by magnetic water and chemical substances during 2014-2015 and 2015-2016 seasons(1).

\begin{tabular}{|c|c|c|c|c|c|c|c|}
\hline \multicolumn{2}{|c|}{ Treatments } & $\begin{array}{l}\text { Bulb di- } \\
\text { ameter } \\
(\mathrm{cm})\end{array}$ & $\begin{array}{l}\text { Neck di- } \\
\text { ameter } \\
\text { (cm) }\end{array}$ & $\begin{array}{c}\text { Plant } \\
\text { height } \\
\text { (cm) }\end{array}$ & $\begin{array}{c}\text { Plant } \\
\text { weight } \\
\text { (g) }\end{array}$ & $\begin{array}{c}\text { Bulbing } \\
\text { ratio } \\
(\mathrm{cm})\end{array}$ & $\begin{array}{c}\text { No. of } \\
\text { leaves /pl. }\end{array}$ \\
\hline & & \multicolumn{6}{|c|}{ 2014-2015 } \\
\hline \multicolumn{2}{|l|}{ "48,(1.5 cm/l) } & $3.72 \mathrm{a}$ & $1.68 \mathrm{~d}$ & $61.00 \mathrm{a}$ & $144.030 \mathrm{~b}$ & $0.451 \mathrm{~d}$ & $12.90 \mathrm{c}$ \\
\hline \multicolumn{2}{|l|}{$48,(3 \mathrm{~cm} / \mathrm{l})$} & $3.18 \mathrm{~b}$ & $1.48 \mathrm{f}$ & $62.6 \mathrm{a}$ & $151.400 \mathrm{a}$ & $0.466 \mathrm{~d}$ & $15.00 \mathrm{a}$ \\
\hline \multicolumn{2}{|l|}{ ** $50,(1.5 \mathrm{~cm} / \mathrm{l})$} & $3.13 \mathrm{~b}$ & $1.80 \mathrm{~b}$ & $55.20 \mathrm{~b}$ & $134.400 \mathrm{~d}$ & $0.575 \mathrm{~b}$ & $11.80 \mathrm{~d}$ \\
\hline \multicolumn{2}{|l|}{$50,(3 \mathrm{~cm} / \mathrm{l})$} & $3.23 \mathrm{~b}$ & $1.72 \mathrm{c}$ & $55.33 \mathrm{~b}$ & $136.860 \mathrm{~cd}$ & $0.535 \mathrm{c}$ & $12.50 \mathrm{dc}$ \\
\hline \multicolumn{2}{|l|}{${ }^{* * * *} \mathbf{M} \mathbf{w}$} & $3.66 \mathrm{a}$ & $1.58 \mathrm{e}$ & $61.50 \mathrm{a}$ & $148.630 \mathrm{a}$ & $0.432 \mathrm{~d}$ & $14.03 \mathrm{~b}$ \\
\hline \multicolumn{2}{|l|}{ Control } & $2.83 \mathrm{c}$ & $1.88 \mathrm{a}$ & $56.23 \mathrm{~b}$ & $139.167 \mathrm{c}$ & $0.664 \mathrm{a}$ & $12.86 \mathrm{c}$ \\
\hline & & \multicolumn{6}{|c|}{ 2015-2016 } \\
\hline \multicolumn{2}{|l|}{$48,(1.5 \mathrm{~cm} / \mathrm{l})$} & $3.40 \mathrm{ab}$ & $1.65 \mathrm{~d}$ & $62.03 \mathrm{a}$ & $144.200 \mathrm{c}$ & $0.494 \mathrm{c}$ & $13.03 \mathrm{c}$ \\
\hline \multicolumn{2}{|l|}{$48,(3 \mathrm{~cm} / \mathrm{l})$} & $3.21 \mathrm{bc}$ & $1.46 \mathrm{f}$ & $62.06 \mathrm{a}$ & $151.733 \mathrm{a}$ & $0.454 \mathrm{dc}$ & $15.00 \mathrm{a}$ \\
\hline \multicolumn{2}{|l|}{$50,(1.5 \mathrm{~cm} / \mathrm{l})$} & $3.14 \mathrm{bc}$ & $1.78 \mathrm{~b}$ & $55.26 \mathrm{~b}$ & $134.267 \mathrm{e}$ & $0.566 \mathrm{~b}$ & $12.20 \mathrm{~d}$ \\
\hline \multicolumn{2}{|l|}{$50,(3 \mathrm{~cm} / \mathrm{l})$} & $3.34 \mathrm{ab}$ & $1.69 \mathrm{c}$ & $55.20 \mathrm{~b}$ & $139.800 \mathrm{~d}$ & $0.507 \mathrm{bc}$ & $12.60 \mathrm{~cd}$ \\
\hline \multicolumn{2}{|l|}{$\mathbf{M} \mathbf{w}$} & $3.67 \mathrm{a}$ & $1.57 \mathrm{e}$ & $62.13 \mathrm{a}$ & $147.633 \mathrm{~b}$ & $0.429 \mathrm{~d}$ & $14.00 \mathrm{~b}$ \\
\hline \multicolumn{2}{|l|}{ Control } & $2.86 \mathrm{c}$ & $1.87 \mathrm{a}$ & $56.06 \mathrm{~b}$ & $140.400 \mathrm{~d}$ & $0.654 \mathrm{a}$ & $12.30 \mathrm{dc}$ \\
\hline $\begin{array}{l}\text { Source of } \\
\text { variation }\end{array}$ & d.f. & \multicolumn{6}{|c|}{ Mean Square } \\
\hline Year & 1 & 0.00360 & 0.00302500 & 0.9025000 & 3.12111 & 0.00007803 & 0.00027778 \\
\hline $\begin{array}{l}\text { Rep(within } \\
\text { year) }\end{array}$ & 4 & 0.034063 & 0.00045556 & 2.9672222 & 4.926111 & 0.00104489 & 0.4211111 \\
\hline Treat & 5 & $0.53906^{*}$ & $0.12628944 *$ & $77.971611^{*}$ & $249.26511^{*}$ & $0.0430413^{*}$ & $7.443611^{*}$ \\
\hline Treat $\times$ year & 5 & $0.03349^{*}$ & $\begin{array}{c}0.00011167 \\
\text { (N.S.) }\end{array}$ & $\begin{array}{c}0.3405000 \\
\text { (N.S.) }\end{array}$ & $2.760444 *$ & $0.00088369 *$ & $0.1529444 *$ \\
\hline Error & 20 & 0.03204 & 0.00026556 & 1.4958889 & 3.070111 & 0.00084919 & 0.17077778 \\
\hline
\end{tabular}

${ }^{(1)}$ Means within column followed by the same letter(s) are not significantly different at 0.05 level of probability.

*Agro promotor1 (substance No.48).

** Agro promotor2 (substance No.50).

${ }^{* * *}$ MW (Magnetic water) 
Table 3. Growth parameters after 90 days from applying treatments (third sample) as affected by magnetic water and chemical substances during 2014-2015 and 2015-2016 seasons ${ }^{(1)}$.

\begin{tabular}{|c|c|c|c|c|c|c|c|}
\hline \multicolumn{2}{|c|}{ Treatments } & $\begin{array}{l}\text { Bulb di- } \\
\text { ameter } \\
\text { (cm) }\end{array}$ & $\begin{array}{l}\text { Neck di- } \\
\text { ameter } \\
\text { (cm) }\end{array}$ & $\begin{array}{c}\text { Plant } \\
\text { height } \\
\text { (cm) }\end{array}$ & $\begin{array}{c}\text { Plant } \\
\text { weight } \\
\text { (g) }\end{array}$ & $\begin{array}{c}\text { Bulbing } \\
\text { ratio } \\
\text { (cm) }\end{array}$ & $\begin{array}{l}\text { No. of } \\
\text { leaves } / p l \text {. }\end{array}$ \\
\hline & & \multicolumn{6}{|c|}{$2014-2015$} \\
\hline \multicolumn{2}{|c|}{ "48,(1.5 cm/l) } & $4.20 \mathrm{~b}$ & $2.33 \mathrm{~b}$ & $71.46 \mathrm{~b}$ & $250.800 \mathrm{c}$ & $0.555 \mathrm{~b}$ & $14.00 \mathrm{c}$ \\
\hline \multicolumn{2}{|c|}{$48,(3 \mathrm{~cm} / \mathrm{l})$} & $4.77 \mathrm{a}$ & $1.97 \mathrm{c}$ & $74.33 \mathrm{a}$ & $318.730 \mathrm{a}$ & $0.413 \mathrm{c}$ & $16.96 \mathrm{a}$ \\
\hline \multicolumn{2}{|c|}{ "**50,(1.5cm/l) } & $4.15 \mathrm{c}$ & $2.40 \mathrm{ab}$ & $64.23 \mathrm{c}$ & $230.330 \mathrm{f}$ & $0.576 \mathrm{~b}$ & $12.93 \mathrm{~d}$ \\
\hline \multicolumn{2}{|c|}{$50,(3 \mathrm{~cm} / \mathrm{l})$} & $4.40 \mathrm{~b}$ & $2.36 \mathrm{ab}$ & $65.46 \mathrm{c}$ & $234.133 \mathrm{e}$ & $0.536 \mathrm{~b}$ & $14.50 \mathrm{c}$ \\
\hline \multicolumn{2}{|l|}{${ }^{* * * *} \mathbf{M ~ w}$} & $4.76 \mathrm{a}$ & $2.00 \mathrm{c}$ & $70.46 \mathrm{~b}$ & $258.500 \mathrm{~b}$ & $0.420 \mathrm{c}$ & $15.96 \mathrm{~b}$ \\
\hline \multicolumn{2}{|l|}{ Control } & $3.54 \mathrm{~d}$ & $2.55 \mathrm{a}$ & $65.53 \mathrm{c}$ & $243.000 \mathrm{~d}$ & $0.720 \mathrm{a}$ & $13.76 \mathrm{dc}$ \\
\hline & & \multicolumn{6}{|c|}{$2015-2016$} \\
\hline \multicolumn{2}{|c|}{$48,(1.5 \mathrm{~cm} / \mathrm{l})$} & $4.13 \mathrm{c}$ & $2.33 \mathrm{~b}$ & $70.46 \mathrm{ab}$ & $253.267 \mathrm{c}$ & $0.564 \mathrm{~b}$ & $13.93 \mathrm{c}$ \\
\hline \multicolumn{2}{|c|}{$48,(3 \mathrm{~cm} / \mathrm{l})$} & $4.70 \mathrm{a}$ & $1.96 \mathrm{c}$ & $74.30 \mathrm{a}$ & $322.90 \mathrm{a}$ & $0.419 \mathrm{c}$ & $16.60 \mathrm{a}$ \\
\hline \multicolumn{2}{|c|}{$50,(1.5 \mathrm{~cm} / \mathrm{l})$} & $4.17 \mathrm{bc}$ & $2.44 \mathrm{ab}$ & $61.56 \mathrm{c}$ & $232.500 \mathrm{e}$ & $0.584 \mathrm{~b}$ & $12.66 \mathrm{~d}$ \\
\hline \multicolumn{2}{|c|}{$50,(3 \mathrm{~cm} / \mathrm{l})$} & $4.35 \mathrm{~b}$ & $2.40 \mathrm{ab}$ & $65.76 \mathrm{bc}$ & $241.00 \mathrm{~d}$ & $0.551 \mathrm{~b}$ & $14.06 \mathrm{c}$ \\
\hline \multicolumn{2}{|l|}{$\mathbf{M} \mathbf{w}$} & $4.77 \mathrm{a}$ & $2.10 \mathrm{c}$ & $70.73 \mathrm{a}$ & $259.83 \mathrm{~b}$ & $0.440 \mathrm{c}$ & $15.16 \mathrm{~b}$ \\
\hline \multicolumn{2}{|l|}{ Control } & $3.56 \mathrm{~d}$ & $2.55 \mathrm{a}$ & $65.00 \mathrm{c}$ & $244.700 \mathrm{~d}$ & $0.715 \mathrm{a}$ & $13.10 \mathrm{~d}$ \\
\hline $\begin{array}{l}\text { Source of } \\
\text { variation }\end{array}$ & d.f & \multicolumn{6}{|c|}{ Mean Square } \\
\hline Year & 1 & 0.004011 & 0.008100 & 3.361111 & 87.42250 & 0.000693 & 1.690000 \\
\hline $\begin{array}{l}\text { Rep } \\
\text { (within } \\
\text { year) }\end{array}$ & 4 & 0.003011 & 0.00171667 & 1.991111 & 14.16333 & 0.00020911 & 0.41805556 \\
\hline Treat & 5 & $0.211211 *$ & $0.30815333^{*}$ & 112.99177* & $6366.79117 *$ & $0.073435^{*}$ & 12.847111* \\
\hline $\begin{array}{l}\text { Treat } \\
\times \text { year }\end{array}$ & 5 & $\begin{array}{c}0.002944 \\
\text { (N.S.) }\end{array}$ & $\begin{array}{c}0.00242667 \\
\text { (N.S.) }\end{array}$ & $\begin{array}{c}1.895111 \\
\text { (N.S.) }\end{array}$ & $6.50317^{*}$ & $\begin{array}{c}0.00011224 \\
\text { (N.S.) }\end{array}$ & $\begin{array}{c}0.10666667 \\
\text { (N.S.) }\end{array}$ \\
\hline Error & 20 & 0.013464 & 0.01325667 & 4.2131111 & 5.47600 & 0.0009777 & 0.19005556 \\
\hline
\end{tabular}


Table 4. Yield and its component as affected by magnetic water and chemical substances during 2014-2015 and 2015-2016 seasons $^{(1)}$.

\begin{tabular}{|c|c|c|c|c|c|c|c|c|c|}
\hline \multicolumn{2}{|c|}{ Treatments } & $\begin{array}{c}\text { Bolters } \\
(\%))\end{array}$ & $\begin{array}{c}\text { Bulb } \\
\text { weight } \\
\text { (g) }\end{array}$ & $\begin{array}{c}\text { Bulb } \\
\text { diameter } \\
(\mathbf{c m})\end{array}$ & $\begin{array}{c}\text { Bulb neck } \\
\text { diameter } \\
\text { (cm) }\end{array}$ & $\begin{array}{c}\text { Bulbing } \\
\text { ratio }\end{array}$ & $\begin{array}{c}\text { marketable } \\
\text { yield } \\
(\%)\end{array}$ & $\begin{array}{c}\text { Total } \\
\text { yield } \\
\text { (ton/fed) }\end{array}$ & TSS \\
\hline & & \multicolumn{8}{|c|}{ 2014-2015 } \\
\hline \multicolumn{2}{|c|}{$48,(1.5 \mathrm{~cm} / \mathrm{l})$} & $23.60 \mathrm{c}$ & $125.33 \mathrm{~d}$ & $5.70 \mathrm{a}$ & $0.633 \mathrm{~d}$ & $0.110 \mathrm{~d}$ & $70.0 \mathrm{c}$ & $9.016 \mathrm{a}$ & $13.0 \mathrm{ab}$ \\
\hline \multicolumn{2}{|c|}{$48,(3 \mathrm{~cm} / \mathrm{l})$} & $19.23 \mathrm{~d}$ & $145.00 \mathrm{~b}$ & $5.40 \mathrm{~b}$ & $0.823 \mathrm{c}$ & $0.152 \mathrm{c}$ & $74.76 \mathrm{~b}$ & $9.166 \mathrm{a}$ & $13.26 \mathrm{a}$ \\
\hline \multicolumn{2}{|c|}{$50,(1.5 \mathrm{~cm} / \mathrm{l})$} & $26.27 \mathrm{~b}$ & $122.00 \mathrm{~d}$ & $5.10 \mathrm{c}$ & $0.817 \mathrm{c}$ & $0.159 \mathrm{bc}$ & $64.33 \mathrm{e}$ & $8.400 \mathrm{~b}$ & $12.86 \mathrm{ab}$ \\
\hline \multicolumn{2}{|c|}{$50,(3 \mathrm{~cm} / \mathrm{l})$} & $23.80 \mathrm{c}$ & $135.00 \mathrm{c}$ & $5.40 \mathrm{~b}$ & $0.893 \mathrm{~b}$ & $0.165 \mathrm{~b}$ & $68.0 \mathrm{~d}$ & $8.500 \mathrm{~b}$ & $12.70 \mathrm{~b}$ \\
\hline \multicolumn{2}{|l|}{${ }^{* x+k} \mathbf{M ~ w}$} & $12.07 \mathrm{e}$ & $149.66 \mathrm{a}$ & $5.53 \mathrm{~b}$ & $0.907 \mathrm{~b}$ & $0.163 \mathrm{~b}$ & $78.5 \mathrm{a}$ & $9.000 \mathrm{a}$ & $13.0 \mathrm{ab}$ \\
\hline \multicolumn{2}{|l|}{ Control } & $27.70 \mathrm{a}$ & $76.00 \mathrm{e}$ & $4.52 \mathrm{~d}$ & $1.023 \mathrm{a}$ & $0.226 \mathrm{a}$ & $60.33 \mathrm{f}$ & $8.030 \mathrm{c}$ & $11.73 \mathrm{c}$ \\
\hline \multicolumn{10}{|c|}{$2015-2016$} \\
\hline \multicolumn{2}{|c|}{$48,(1.5 \mathrm{~cm} / \mathrm{l})$} & $23.30 \mathrm{c}$ & $125.900 \mathrm{~d}$ & $5.76 \mathrm{a}$ & $0.650 \mathrm{c}$ & $0.112 \mathrm{c}$ & $71.2 \mathrm{c}$ & $9.166 \mathrm{a}$ & $13.13 \mathrm{a}$ \\
\hline \multicolumn{2}{|c|}{$48,(3 \mathrm{~cm} / \mathrm{l})$} & $19.50 \mathrm{~d}$ & $147.433 \mathrm{~b}$ & $5.56 \mathrm{a}$ & $0.850 \mathrm{~b}$ & $0.152 \mathrm{~b}$ & $75.16 \mathrm{~b}$ & $9.250 \mathrm{a}$ & $13.23 \mathrm{a}$ \\
\hline \multicolumn{2}{|c|}{$50,(1.5 \mathrm{~cm} / \mathrm{l})$} & $25.83 \mathrm{~b}$ & $122.800 \mathrm{~d}$ & $5.10 \mathrm{~b}$ & $0.816 \mathrm{~b}$ & $0.160 \mathrm{~b}$ & $66.20 \mathrm{e}$ & $8.416 \mathrm{c}$ & $12.60 \mathrm{~b}$ \\
\hline \multicolumn{2}{|c|}{$50,(3 \mathrm{~cm} / \mathrm{l})$} & $22.93 \mathrm{c}$ & $134.333 \mathrm{c}$ & $5.60 \mathrm{a}$ & $0.873 \mathrm{~b}$ & $0.155 \mathrm{~b}$ & $70.03 \mathrm{~d}$ & $8.550 \mathrm{~b}$ & $12.70 \mathrm{~b}$ \\
\hline \multirow{2}{*}{\multicolumn{2}{|c|}{$\begin{array}{l}\text { M w } \\
\text { Control }\end{array}$}} & $11.93 \mathrm{e}$ & $151.700 \mathrm{a}$ & $5.60 \mathrm{a}$ & $0.866 \mathrm{~b}$ & $0.154 \mathrm{~b}$ & $80.0 \mathrm{a}$ & $9.250 \mathrm{a}$ & $13.17 \mathrm{a}$ \\
\hline & & $27.86 \mathrm{a}$ & $79.333 \mathrm{e}$ & $4.66 \mathrm{c}$ & $1.006 \mathrm{a}$ & $0.215 \mathrm{a}$ & $61.43 \mathrm{f}$ & $8.016 \mathrm{~d}$ & $11.60 \mathrm{c}$ \\
\hline \multicolumn{2}{|l|}{ Source of } & \multicolumn{8}{|c|}{ Mean Square } \\
\hline Year & 1 & 0.42250 & 18.06250 & 0.10454444 & 0.00027778 & 0.000191 & 16.2677 & 0.07111 & 0.00444 \\
\hline $\begin{array}{l}\text { Rep } \\
\text { (within } \\
\text { year) }\end{array}$ & 4 & 0.09055 & 7.69667 & 0.00837778 & 0.00021389 & 0.000005 & 0.3752 & 0.02694 & 0.0513888 \\
\hline Treat & 5 & $192.90^{*}$ & 4130.459* & $1.0400111^{*}$ & $0.0891666^{*}$ & $0.00734^{*}$ & $260.4664 *$ & $1.3897^{*}$ & 1.96533* \\
\hline $\begin{array}{l}\text { Treat } \times \\
\text { year }\end{array}$ & 5 & $0.2591^{*}$ & $3.15917 *$ & $0.0085444^{*}$ & $0.0009244^{*}$ & $0.00004 *$ & $0.526444^{*}$ & $0.0142 *$ & $0.039777^{*}$ \\
\hline Error & 20 & 0.20822 & 4.18433 & 0.01217778 & 0.00114056 & 0.000026 & 0.4532 & 0.01536 & 0.0477222 \\
\hline
\end{tabular}

(1) Means within column followed by the same letter(s) are not significantly different at 0.05 level of probability.

*Agro promotor1 (substance No.48).

** Agro promotor2 (substance No.50).

**** MW (Magnetic water)

\section{Literature Cited}

Amer D. Majeed, Salih M. Salman (2006). A study the Effect of Magnetic Field on the absorption spectrum of Distilled Water, AL-fateh, Journal, No 27,

Ahmed AM. (2013). Effects of Magnetized Low Quality Water on Some Soil Properties and Plant Growth, Int. J. Res. Chem. Environ. 3:14014.

Basant L. Maheshwari, Harsharn Singh Grewal. (2009). Magnetic treatment of irrigation water: Its effects on vegetable crop yield and water productivity, Agricultural Water Management 96, 1229-1236, www.elsevier.com/locate/agwa.

Belyavskaya, N.A. (2001). Ultrastructure and calcium balance in meristem cells of pea roots exposed to ex- tremely low magnetic field. Adv. Space Res., 28: 4, 645-650.

Carbonell, M.V., Florez, M., Martinez E., Maqueda, R. and J.M. Amaya (2011). Study of stationary magnetic fields on initial growth of pea (Pisumsativum L.) seeds. Seed Science and Technology, 39: 673679.

Esitken, A. (2003). Effects of magnetic fields on yield and growth in strawberry 'Camarosa'. Journal of Horticultural Science \& Biotechnology, 78 (2): 145- 147.

FAOSTAT, (2010). An on-line and multilingual databases currently containing over one million timeseries records covering international statistics.

Formicheva, V.M, Govorun, R.D and V.T. Danilov (1992a). Proliferative 
activity and cell reproduction in the root meristem of pea, lentil and flax in the conditions of screening the geomagnetic field. Int. Biophysics. 37: 645-648.

Formicheva, V.M, Zaslavskii, V.A, Govorun, R.D. and V.T. Danilov (1992b). Dynamics of RNA and protein synthesis in the cells of the root meristems of the pea, lentil and flax Biophysics. 37: 649-656.

Gholizadeh, M. and H. Arabshahi, (2008). The effect of magnetic water on growth and quality improvement of poultry. Middle-East Journal of Scientific Research, 3.

Hassan, A. A. (1991). Production of vegetable crops. $1^{\text {st }}$ ed., Published by Arab House for Publishing and Distribution, Cairo, Egypt. (In Arabic).

Hozayn M., and Mohamed A. and S. Abdul Qados (2010). Irrigation with magnetized water enhances growth, chemical constituent and yield of chickpea (Cicerarietinum L.). AGRICULTURE AND BIOLOGY JOURNAL OF NORTH AMERICA. ISSN Print: 21517517, ISSN Online: 2151-7525.

Kaoud, H.A. (2014).'Patent" Agropromotor 1 and 2. Fac.vet. Cairo university- Egypt.

L. Kordas (2002). The effect of magnetic field on growth, development and the yield of spring wheat, Polish Journal of Environmental Studies Vol. 11, 527-530. No. 5.

Lin, I. and J. Yotvat, (1990). Exposure of irrigation and drinking water to a magnetic field with controlled power and direction. Journal of magnetism and magnetic materials, 83(1): p. 525-526 (1990).

Mann,L.K. (1952). Anatomy of the garlic bulb development. Hilgardia, 21:195-228.

Özalpan A, Atak C, Yurttas B, Alikamanoglu S, Canbolat Y, Borucu H, Danilov V, Rzakoulieva A (1999). Effect of magnetic field on soybean yield (Glycine max L. Merrill). Turkish Association of Biophysics, XI National Biophys- ics Congress, Abstract Book. 1999;60.

Oldacay S, Erdem G (2002). Evaluation of chlorophyll contents and peroxides activities in I (Helianthus annuus L.) genotypes exposed to radiation and magnetic field. Pak. J. of Appl. Sci. 2(10):934-937.

Osman E.A. M., Abd El-Latif K.M, Hussien S.M. and A.E.A. Sherif (2014). Assessing the effect of irrigation with different levels of saline magnetic water on growth parameters and mineral contents of pear seedlings. Glob. J. Sci. Res., 2 (5): 128-136, 2014.

Rawabdeh, H., Safwan S. and S. Rida (2014). The Effect of Irrigation by Magnetically Water on Chlorophyll and Macroelements uptake of Pepper (Capsicum annuum L.). Jordan Journal of Agricultural Sciences, Volume 10, No.2.

Samir.H.Nasher, (2008). The effect of magnetic water on growth of Chick-Pea seeds, Eng. \& Tech. Vol. 26, No.9.

Snedecor, C.W. and W.G. Cochran. (1980). Statistical methods. $6^{\text {th }}$ ed. Iowa Univ. Press, Ames, Iowa, U.S.A.

Yano, A., Ohashi, Y., Hirasaki, T. and K. Fujiwara (2004). Effects of 60 $\mathrm{Hz}$ magnetic field on photosynthetic uptake and early growth of radish seedlings. Bioelectromagnetics, 25(8): 572-581.

Yadollahpour, A., Rashidi, S., and K.Fatemeh (2014). Applications of Magnetic Water Technology in Farming and Agriculture Development: A Review of Recent Advances. Current World Environment Vol. 9(3), 695-703.

Yurttas B, Atak C, Gökdoan G, Canbolat Y, Danilov V, Rzakoulieva A(1999). Detection of the positive effect of magnetic field on sunflower plants (Helianthus annuus L.). Turkish Association of Biophysics, XI National Biophysics Congress, Abstract Book. 1999;59. 
تأثثير الماء الممغنط والمواد الكيميائية على صفات النمو، المحصول ومكوناتة فى محصول البصل تحت ظروف اسيوط النو، شرين يعقوب عطالله

قسم الخضر - كلية الزر اعة - جامعة اسيوط

الملخص

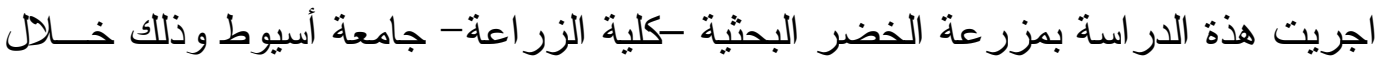

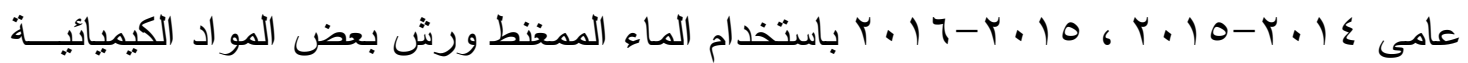

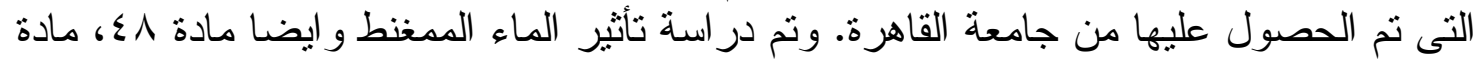

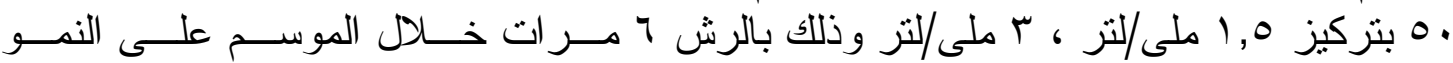

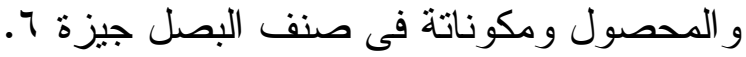

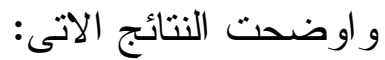

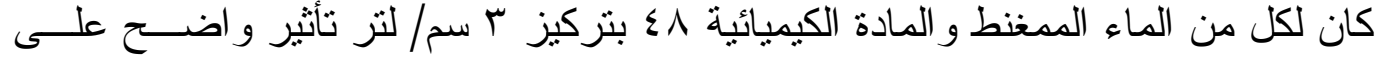

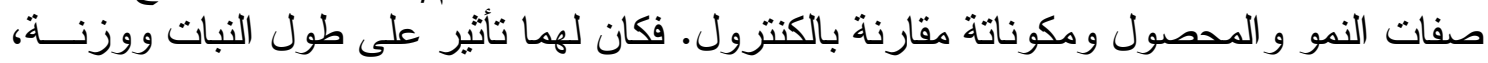

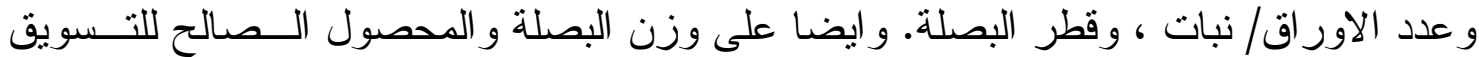

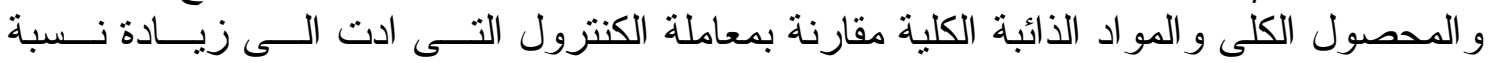

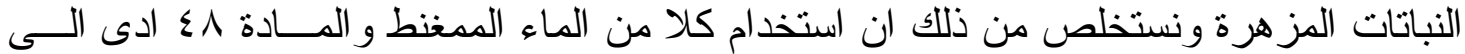

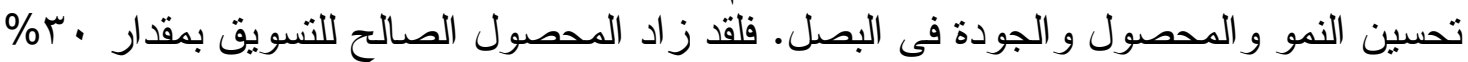

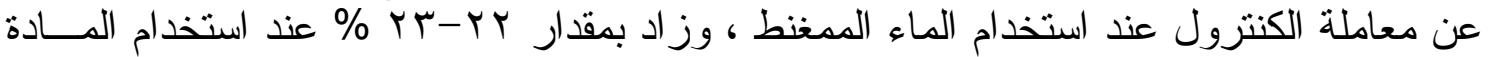

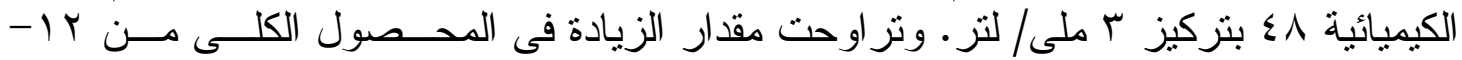

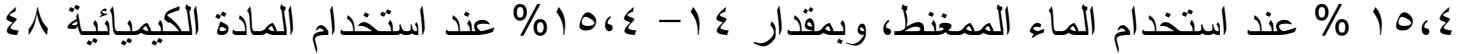

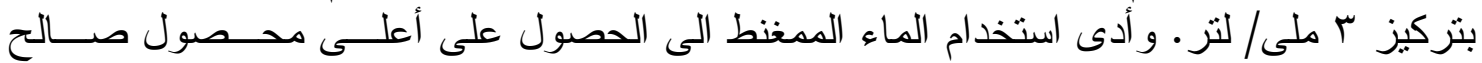

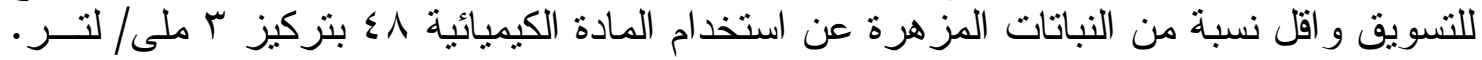

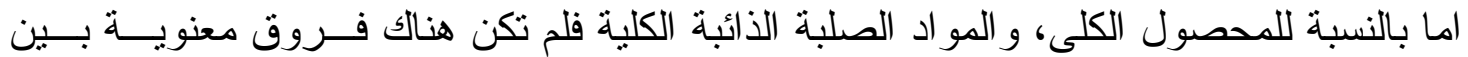

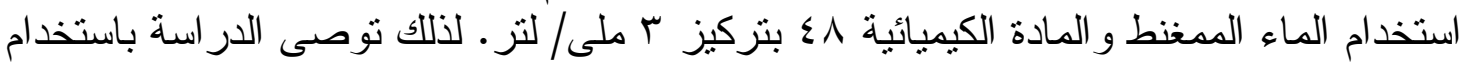

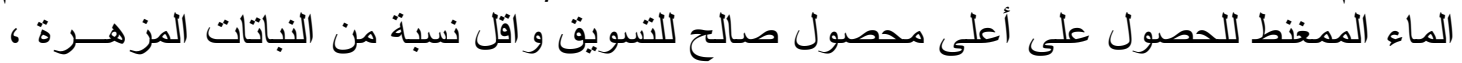

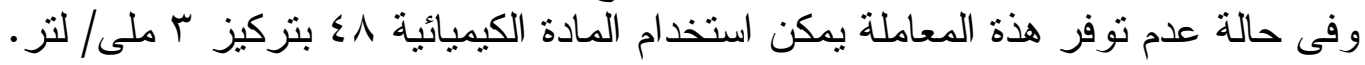

